\title{
How to Treat Multiple Myeloma: Thoughts on a Multicentre Survey in Germany
}

\author{
Joan Bladé Laura Rosiñol $\quad \mathrm{M}^{\mathrm{a}}$ Teresa Cibeira Carlos Fernández de Larrea
}

Department of Hematology, Hospital Clínic, Barcelona, Institut d'Investigacions Biomèdiques August Pi I Sunyer (IDIBAPS), University of Barcelona, Barcelona, Spain

Knauf et al. [1] report in the current issue of ONKOLOGIE the results of a multicenter survey study performed in order to learn on the changes in the management of multiple myeloma (MM) over time in Germany. For this purpose 386 patients diagnosed in a 3 months-period from 35 centres in Germany (14\% university hospitals, $46 \%$ community hospitals and $40 \%$ office-based haematologists) were studied and the results were compared with similar surveys from 2004 and 2006. The authors concluded that: i) there were important deviations from international recommended guidelines [2], ii) a high proportion of patients in Durie and Salmon stage I were treated, iii) a minority of patients was considered for highdose therapy/stem cell transplantation (HDT/SCT), and iv) a shift towards the incorporation of novel drugs in the treatment of MM. Although this study demonstrates an improvement when compared with the results from 2004 and 2006, there are issues that remain of concern in the general management of patients with MM.

First, from this study it seems evident that the baseline evaluation is still suboptimal. Thus, $\beta_{2}$-microglobulin and serum albumin, two easily available parameters and which are required for the current International Staging System classification [3], were missing in almost $30 \%$ of the patients. Even more striking, FISH cytogenetics, the most important prognostic parameter, was studied in only $22 \%$ of the patients. Surprisingly (or not surprisingly) 'proteinuria' was only measured in $8 \%$ of the patients. A minimal baseline evaluation should include the basic haematological plus chemical values (including haemoglobin level plus serum creatinin, calcium, albumin and $\beta_{2}$-microglobulin) plus total serum protein, 24-h urine protein excretion with serum and urine electrophoresis plus serum and urine immunofixation, skeletal survey and bone marrow aspirate with FISH analysis whenever possible. All myeloma treating physicians should keep in mind that the initial disease assessment, response evaluation and monitoring is not possible without the essential studies on serum and urine basic protein assays $[2,4,5]$.

\section{KARGER}

Fax +497614520714

Information@Karger.de

www.karger.com
(C) 2010 S. Karger GmbH, Freiburg

Accessible online at:

www.karger.com/onk
Second, in the PETHEMA studies the number of patients with Durie and Salmon Stage I myeloma is less than 5\% [6]. The $16.9 \%$ in this study seems too high and the population appears to be heterogeneous. In all myeloma series there is a proportion of patients with symptomatic stage I MM who require systemic therapy from the beginning. However, one must be certain that the patient has not solitary bone plasmacytomas or smouldering (asymptomatic) MM (SMM). In the first case, surgery and/or radiation therapy should be the treatment of choice [2]. In case of SMM, no treatment should be administered until evident progressive or symptomatic myeloma develops [7]. Bisphosphonates are not recommended in SMM [8].

Third, it is concluded that only $35 \%$ of the patients were considered for HDT/SCT. Considering that in the study population only $40 \%$ of the patients were younger that 65 years, the number of patients considered eligible for HDT/SCT seems reasonable. While it is true that fit patients aged $65-70$ years can be eligible for HDT/SCT, there are patients with MM younger than 65 years that are not candidates for HDT (i.e., patients with severe co-morbidities, with poor performance status or disease complications such as advanced renal failure as well as patients with initially unresponsive progressive disease) [9].

Fourth, the increased use of novel agents in the upfront as well as in the relapse/refractory settings in the more recent years is an expected finding and should be encouraged [10]. Fortunately, we are already in an era of improvement in quality of life and survival of patients with MM and we can foresee an improved long-term outcome for our patients with MM through a number of medical interventions: i) the possible use of a tailored front-line therapy for elderly patients optimising the use of MPT (melphalan, prednisone and thalidomide), MPV (melphalan, prednisone and bortezomib) or lenalidomide/dexamethasone according to age, co-morbidities, performance status and disease aggressiveness, ii) using more effective pre-transplant regimens for younger patients eligible 
for HDT/SCT resulting in a high pre- and post-transplant complete response rate and this leading to a significantly longer survival, iii) selecting the more appropriate rescue regimens after relapse and exploiting the available drugs in a sequential manner according to previous drug exposure, depth and duration of response and previous toxicities, iv) careful baseline and response evaluation with an appropriate monitoring that allows the recognition of clinical progression ensuring a timely rescue therapy, v) adequate prophylaxis and management of toxicities, particularly peripheral neuropathy and thrombotic events, and vi) supportive care with optimal use of biphosphonates, infectious prophylaxis in high-risk patients (renal failure, very high IgG levels with low back- ground immunoglobulins and those with past history of recurrent bacterial infections, particularly pneumococcal pneumonia) appropriate use of erythropoietin as well as an adequate management of renal failure $[11,12]$.

\section{Conflict of Interest}

L.R. and M.T.C. received honoraria for lectures and Advisory Boards from Janssen-Cilag and Celgene; J.B. received honoraria for lectures and Advisory Boards as well as grant support from Janssen-Cilag and Celgene. C.F.d.L. declares no competing financial interests.

\section{References}

1 Knauf W, Kellermann L, Poenisch W, Einsele H, Straka C, Frohn C, GoldschmidtH: How to treat multiple myeloma - a representative multicentre treatment survey. Onkologie 2010;33:604-610.

2 International Myeloma Working Group: Criteria for the classification of monoclonal gammopathies, multiple myeloma and related disorders: a repor of the International Myeloma Working Group. Br J Haematol 2003;121:749-757.

3 Greipp PR, San Miguel J, Durie BG, Crowley JJ, Barlogie B, Bladé J, Boccadoro M, Child JA, Avet-Loiseau H, Kyle RA, Lahuerta JJ, Ludwig H, Morgan G, Powles R, Shimizu K, Shustik C, Sonneveld P, Tosi P, Turesson I, Westin J: International staging system for multiple myeloma. $\mathrm{J}$ Clin Oncol 2005;23:3412-3420.

4 Bladé J, Samson D, Reece D, Apperley J, Björkstrand B, Gahrton G, Gertz M, Giralt S, Jagannath S, Vesole D: Criteria for evaluating disease response and progression in patients with multiple myeloma treated by high-dose therapy and haemopoietic stem cell transplantation. Myeloma Subcommittee of the EBMT. European Group for Blood and Marrow Transplant. Br J Haematol 1998;102:1115-1123.
5 Durie BG, Harousseau JL, Miguel JS, Bladé J, Barlogie B, Anderson K, Gertz M, Dimopoulos M, Westin J, Sonneveld P, Ludwig H, Gahrton G, Beksac M, Crowley J, Belch A, Boccadaro M, Cavo M, Turesson I, Joshua D, Vesole D, Kyle R, Alexanian R, Tricot G, Attal M, Merlini G, Powles R, Richardson P, Shimizu K, Tosi P, Morgan G, Rajkumar SV; International Myeloma Working Group: International uniform response criteria for multiple myeloma. Leukemia 2006;20:1467-1473.

6 Bladé J, San Miguel JF, Alcalá A, Maldonado J, Sanz MA, García-Conde J, Moro MJ, Alonso C, Besalduch J, Zubizarreta A: Alternating combination VCMP/VBAP chemotherapy versus melphalan/prednisone in the treatment of multiple myeloma: a randomized multicentric study of 487 patients. J Clin Oncol 1993;11:1165-1171.

7 Bladé J, Dimopoulos M, Rosiñol L, Rajkumar SV, Kyle RA: Smoldering (asymptomatic) multiple myeloma: current diagnostic criteria, new predictors of outcome, and follow-up recommendations. J Clin Oncol 201;28:690-697.
8 Terpos E, Sezer O, Croucher PI, García-Sanz R, Boccadoro M, San Miguel J, Ashcroft J, Bladé J, Cavo M, Delforge M, Dimopoulos MA, Facon T, Macro M, Waage A, Sonneveld P; European Myeloma Network: The use of bisphosphonates in multiple myeloma: recommendations of an expert panel on behalf of the European Myeloma Network. Ann Oncol 2009;20:1303-1317.

9 Bladé J, Rosiñol L, Cibeira MT, Rovira M, Carreras E: Hematopoietic stem cell transplantation for multiple myeloma beyond 2010. Blood 2010;115:3655-3663.

10 Bladé J, Rosiñol L: Changing paradigms in the treatment of multiple myeloma. Haematologica 2009;94:163-166.

11 Bladé J, Rosiñol L: Complications of multiple myeloma. Hematol Oncol Clin North Am 2007; 21:1231-1246.

12 Terpos E, Cibeira MT, Blade J, Ludwig H: Management of complications in multiple myeloma. Semin Hematol 2009;46:176-189. 